\title{
Spiral Honeycomb Microstructured Bacterial Cellulose for Increased Strength and Toughness
}

\author{
Kui Yu, Srikkanth Balasubramanian, Helda Pahlavani, Mohammad J. Mirzaali, Amir A. Zadpoor,
} and Marie-Eve Aubin-Tam*

Cite This: ACS Appl. Mater. Interfaces 2020, 12, 50748-50755

Read Online

ABSTRACT: Natural materials, such as nacre and silk, exhibit both high strength and toughness due to their hierarchical structures highly organized at the nano-, micro-, and macroscales. Bacterial cellulose (BC) presents a hierarchical fibril structure at the nanoscale. At the microscale, however, $\mathrm{BC}$ nanofibers are distributed randomly. Here, BC self-assembles into a highly organized spiral honeycomb microstructure giving rise to a high tensile strength $(315 \mathrm{MPa})$ and a high toughness value $(17.8 \mathrm{MJ}$ $\mathrm{m}^{-3}$ ), with pull-out and de-spiral morphologies observed during failure. Both experiments and finite-element simulations indicate
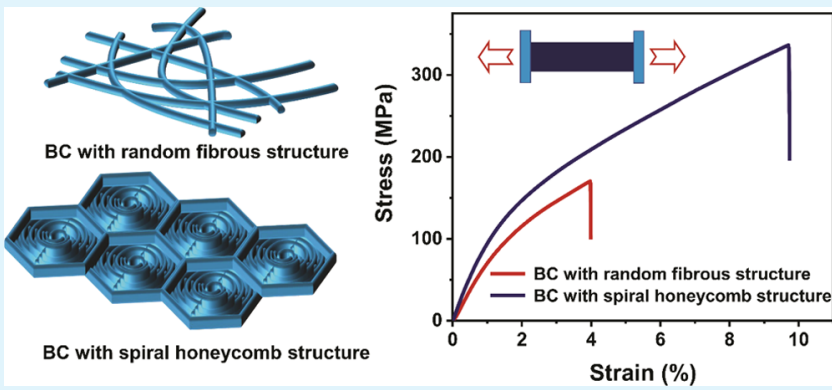
improved mechanical properties resulting from the honeycomb structure. The mild fabrication process consists of an in situ fermentation step utilizing poly(vinyl alcohol), followed by a posttreatment including freezing-thawing and boiling. This simple self-assembly production process is highly scalable, does not require any toxic chemicals, and enables the fabrication of light, strong, and tough hierarchical composite materials with tunable shape and size.

KEYWORDS: bio-inspired materials, cellular materials, sustainability, self-assembly, biocomposites

\section{INTRODUCTION}

High-performance materials that have lightweight, high strength, and high toughness are highly demanded in the aerospace, biomedical, and construction industries. However, strength and toughness are generally considered mutually exclusive properties in artificial materials. ${ }^{1}$ In biological systems, on the other hand, there are multiple examples of strong and tough materials (e.g., spider silk, ${ }^{2}$ nacre, ${ }^{3}$ bone, ${ }^{4}$ and $\left.\operatorname{wood}^{5}\right)$. This is achieved via their hierarchical structure, which is highly ordered from the molecular and microscale up to the macroscale. ${ }^{6}$ The construction of highly organized hierarchical structures is key to produce high-performance structural materials. ${ }^{7,8}$

To achieve such hierarchical structures, different types of materials could be used, including organic materials (e.g., polymers, ${ }^{9}$ carbon-based ${ }^{10}$ ), inorganic materials (e.g., calcium carbonate $^{11}$ ), or a combination of both. ${ }^{12}$ Among those materials, natural biopolymers are drawing increasing attention due to their bio-inspired nature, current environmental concerns, and the need for sustainable materials. ${ }^{9}$ Cellulose is a particularly interesting example of such green biopolymers. As the most abundant biopolymer in nature, cellulose, in the form of nanofibers, widely exists in most plants and wood structures, ${ }^{13}$ as well as in the biofilms surrounding some microorganisms (e.g., in Gluconacetobacter hansenii). ${ }^{14}$ Cellulose nanofibers themselves consist of a hierarchical fibril structure originating from the strong intramolecular and intermolecular hydrogen bonding, resulting in high tensile strength and elastic modulus. ${ }^{13}$ Therefore, cellulose nanofibers are ideal building blocks for constructing high-performance materials with organized mesoscale structures. ${ }^{15}$ In particular, bacterial cellulose (BC) is secreted in large quantities by bacteria in the form of a hydrogel-like biofilm. ${ }^{16}$ This biofilm consists of randomly distributed single BC nanofibers, ${ }^{17}$ which possess the same organized fibril structure as plant cellulose, ${ }^{18}$ exhibiting high crystallinity and good mechanical performance. ${ }^{19}$ To obtain BC nanofibers, the most frequently used method is to mechanically disintegrate the wet $\mathrm{BC}$ hydrogel pellicles. ${ }^{20}$ These $\mathrm{BC}$ nanofibers are then recombined together using techniques, such as wet spinning ${ }^{20}$ or $3 \mathrm{D}$ printing. ${ }^{21}$ These ex situ methods, however, destroy the naturally layered BC structure and weaken its mechanical performance significantly. ${ }^{22}$ To increase the tensile strength and toughness of $\mathrm{BC}$, an organized $\mathrm{BC}$ microstructure is desired. ${ }^{23}$ Several methods, including wet stretching, ${ }^{18,23}$ twisting, and tape

Received: September 3, 2020

Accepted: October 16, 2020

Published: October 28, 2020 
peeling, ${ }^{24}$ can produce an aligned $\mathrm{BC}$ microstructure based on the natural BC network. These methods could demonstrate that aligning $\mathrm{BC}$ at the microscale enhances the mechanical performance significantly, but they are usually highly energyintensive and show limited scalability.

Here, we report the formation of a highly organized spiral honeycomb microstructure in $\mathrm{BC}$ films via a self-assembly process under mild conditions that combines an in situ fermentation and a post-treatment procedure (Figure 1).

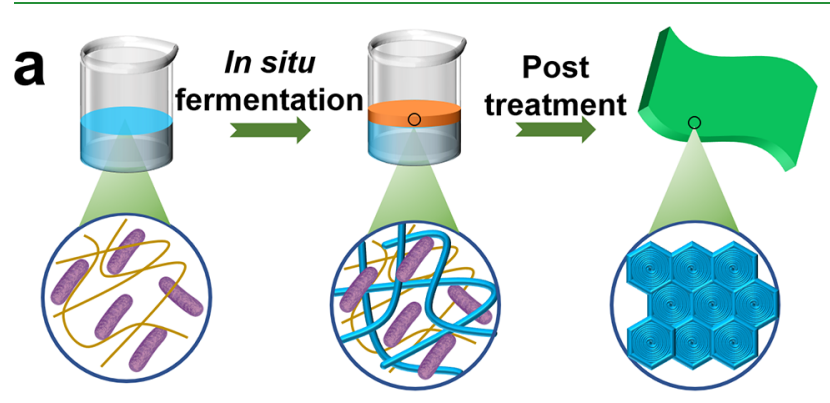

Bacteria PVA polymer Cellulose fibers
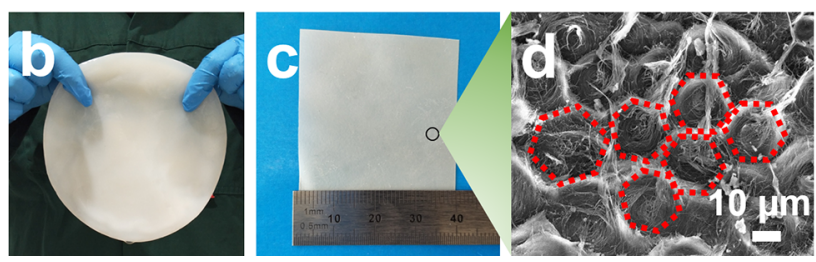

Figure 1. Fabrication and characterization of the self-assembled spiral honeycomb bacterial cellulose film. (a) Illustration of fabrication procedure of $\mathrm{BC}$ film with honeycomb structure. PVA was added to the fermentation medium. The film was formed at the air-liquid interface and was processed with a freezing-thawing, boiling, washing, and drying procedure. (b) Optical image of $\mathrm{BC}$ wet pellicle before air-drying. (c) Optical image of honeycomb BC film. (d) Scanning electron microscope (SEM) image of BC film, showing a spiral honeycomb microstructure.

During the $\mathrm{BC}$ growing procedure, poly(vinyl alcohol) (PVA), a water-soluble additive often used during $\mathrm{BC}$ fermentation to regulate $\mathrm{BC}^{\text {'s biological }}{ }^{25}$ and mechanical property, ${ }^{26}$ is added to the fermentation medium and $\mathrm{BC}$ wet pellicles are harvested at the air-liquid interface. We then apply a freezing-thawing (FT) procedure, followed by boiling, washing, and air-drying. These mild treatments provide a green and scalable alternative to the fabrication of nanofibrous $\mathrm{BC}$ with tailored shapes and sizes, and interestingly, lead to the self-assembly of the material into a spiral honeycomb microstructure. These honeycomb films exhibit higher tensile strength and higher toughness compared to BC films of same composition but without a spiral honeycomb architecture. Hexagonal honeycomb structures are abundant in nature and are capable of adjusting the mechanical performance of various materials. ${ }^{27}$ Furthermore, in honeycomb microstructured samples, nanofibers are densely packed in a spiral form, which is an efficient way to enhance stretchability. $^{28}$

\section{RESULTS AND DISCUSSION}

Biofabrication Procedure and Morphology of Composites. In this work, cellulose nanofibers are produced by G. hansenii, a strain of bacteria with high cellulose production yield. ${ }^{14}$ Cellulose from plant or wood is generally not pure, containing lignin, hemicellulose, pectin, etc., ${ }^{29}$ and an environmentally unfriendly delignification procedure is needed. ${ }^{30}$ On the contrary, $\mathrm{BC}$ consists of pure cellulose without those impurities, ${ }^{31}$ and is therefore an advantageous building block in manufacturing cellulose-based advanced materials. ${ }^{8}$ A straightforward approach to produce BC-based composites is in situ fermentation, ${ }^{32}$ which consists of simply dissolving watersoluble polymers into the fermentation medium during cellulose production by the bacteria. The produced BC nanofibers tend to aggregate at the air-liquid interface, forming a random nanofibrous network held together via strong hydrogen bonding. The liquid medium, including the dissolved polymers, is then entrapped into the $\mathrm{BC}$, resulting in a homogeneous spread of water-soluble polymers into the $\mathrm{BC}$ network. For these polymers to remain in the composites, cross-linking methods are generally used. ${ }^{33}$

Here, the in situ fabrication procedure consists of adding PVA at $10 \% \mathrm{w} / \mathrm{v}$ into the $G$. hansenii fermentation medium. After 10 days of culturing at $30{ }^{\circ} \mathrm{C}, \mathrm{BC} / \mathrm{PVA}$ pellicles are harvested at the air-liquid interface. To cross-link PVA, these pellicles are frozen at $-20{ }^{\circ} \mathrm{C}$ for $24 \mathrm{~h}$ and brought to room temperature to thaw for $6 \mathrm{~h}$. This FT procedure is repeated five times, and then the material is boiled in water for sterilization, immersed in water for washing, and finally dried in air (Figure $1 \mathrm{a}-\mathrm{c}$ ).

Interestingly, a highly organized layered spiral honeycomb structure is observed in these films (Figure $2 \mathrm{a}-\mathrm{f}$ and

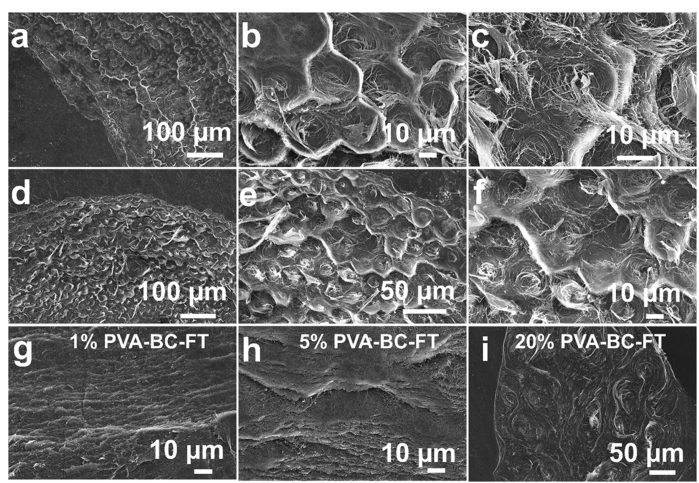

Figure 2. SEM images of the bacterial cellulose films. $(a-c)$ Horizontal sections and $(\mathrm{d}-\mathrm{f})$ cross sections of $10 \%$ PVA-BC-FT composite film. SEM images of the cross section of (g) 1\% PVA-BCFT, (h) 5\% PVA-BC-FT, and (i) 20\% PVA-BC-FT.

Supporting Information Figure S1, 10\% PVA-BC-FT samples). The SEM images of horizontal sections (Figure $2 \mathrm{a}-\mathrm{c}$ ) reveal that $\mathrm{BC}$ nanofibers are spirally and densely packed to form a hexagonal microunit, with borders of these microunits consisting of aligned and entangled BC nanofibers. This results in a honeycomb morphology with spiral BC nanofibers (Figure 2d-f). While the 10\% PVA-BC-FT film keeps the layered structure of natural BC (Supporting Information Figure S2a), the microscale arrangement in each layer is changed from the randomly distributed nanofibers seen in natural BC (Supporting Information Figure S2b) to a spiral honeycomb structure.

To investigate the underlying mechanisms behind the formation of such a structure, we vary the PVA concentration in the fermentation medium between 1 and $20 \% \mathrm{w} / \mathrm{v}$. The sample cross sections show a random fibrous structure for both $1 \% \mathrm{w} / \mathrm{v}$ PVA (1\% PVA-BC-FT, Figure $2 \mathrm{~g}$ ) and 5\% w/v PVA 
(5\% PVA-BC-FT, Figure 2h), as observed in natural BC. However, the samples with $20 \% \mathrm{w} / \mathrm{v}$ PVA (20\% PVA-BC-FT, Figure $2 \mathrm{i}$ and Supporting Information Figure S3) show a spiral fibrous structure. This structure is not identical to the hexagonal spiral honeycomb structure seen in the samples with $10 \% \mathrm{w} / \mathrm{v}$ PVA (10\% PVA-BC-FT). Indeed, the spiral unit diameter increases to $50 \mu \mathrm{m}$ for $20 \%$ PVA-BC-FT compared to $23 \mu \mathrm{m}$ in the case of $10 \%$ PVA-BC-FT. Therefore, the PVA concentration in the initial fermentation medium is of key importance for the formation of the spiral structure. Spirals tend to be formed when the PVA concentration exceeds $10 \% \mathrm{w} / \mathrm{v}$, while $10 \% \mathrm{w} / \mathrm{v}$ is the optimal concentration for the formation of ordered spiral hexagonal structures.

To assess whether PVA concentration is the only factor influencing the structure, the FT was omitted from the fabrication method. The 10\% PVA-BC-FT samples are compared to $10 \%$ PVA-BC, produced following the same procedure except for the FT. Interestingly, there is no honeycomb or spiral structure observed in 10\% PVA-BC (Supporting Information Figure S4). The FT process is therefore essential to the formation of spiral honeycombs. From these results, both the PVA concentration in the fermentation medium and the post-treatment process are crucial factors contributing to the formation of this fibrous honeycomb structure.

The presence of PVA in the honeycomb composites is confirmed with Fourier transform infrared (FTIR) spectra (Supporting Information Figure S5). Thermogravimetry analysis (TGA) reveals that only a small amount of PVA is present in the final honeycomb film since the TGA of pure BC and that of honeycomb composites are similar (Supporting Information Figure S6). PVA in the composites is likely washed away during the post-treatment procedure. During the FT procedure, PVA polymer chains are expected to become more organized and form PVA crystals. ${ }^{34}$ Upon boiling, PVA polymer chains in the crystal likely rearrange and become less organized due to heating, causing the PVA to be water-soluble again. Before this heating procedure, PVA is spread homogeneously in the $\mathrm{BC}$ network and acts as a plasticizer. As the PVA polymer chain is rich in hydrogen bonds, PVA may interfere with the hydrogen bonding between the cellulose fibrils. ${ }^{32}$ The hydrogen bonds in the original BC fibers may, consequently, be weakened, resulting in the rearrangement of the random $\mathrm{BC}$ nanofibers and initiating a self-assembly process that leads to the formation of the spiral honeycomb structure in the post-treatment. Meanwhile, as no honeycomb structure were observed without FT, the PVA crystals during $\mathrm{FT}^{34}$ might act as a template to form the highly ordered structure: the $\mathrm{BC}$ fibers could reorganize into the honeycomb spirals based on the PVA crystal template. During the boiling procedure, this PVA crystal template is removed while the spiral honeycomb structure remains in the composites. This could explain why a higher amount of PVA contributes to the formation of the spiral structure.

Bacterial Viability and Yield of Materials Production. We then assess whether the yield of BC/PVA composite produced depends on the bacterial viability, which can be influenced by the presence of PVA. To study this, the wet thickness of fermented BC is measured after different fermentation times $(5,10$, and 15 days), with and without PVA, and the corresponding amount of viable bacteria is assessed. In all sample types, the bacteria viability reaches a maximum after 5 days of fermentation (Figure 3a). After 5 and 10 days of culture, bacterial viability in pure $\mathrm{BC}$ and $\mathrm{BC} / \mathrm{PVA}$
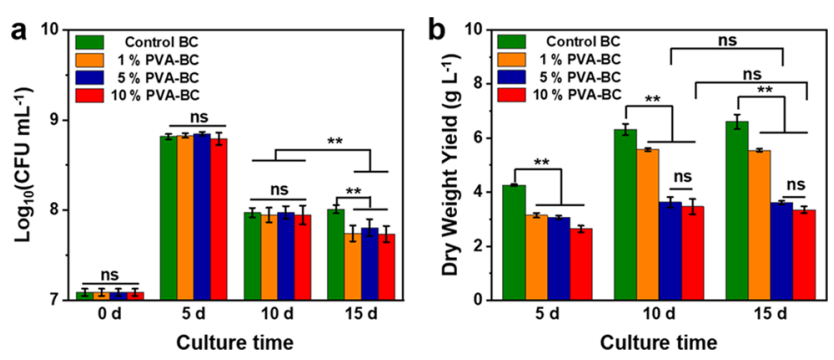

Figure 3. Bacterial viability during fermentation and the yield of the fermented BC/ PVA pellicle. (a) Colony-forming units (CFUs) measurements, (b) dry weight yield. $* * p<0.01$, significant; ns, insignificant.

composites is similar. After 15 days, however, pure BC shows higher bacteria viability. Therefore, the addition of PVA in the fermentation medium lowers the number of viable bacteria only for periods longer than 10 days. The dry weight yield of all specimens remains unchanged after 10 days (Figure $3 \mathrm{~b}$ ) because bacteria entrapment into the $\mathrm{BC}$ pellicle and limited contact with oxygen restricts $\mathrm{BC}$ production after 10 days. ${ }^{35}$ The wet thickness, wet weight, and dry weight measurements (Figure 3b, Supporting Information Figure S7a,b) all show a decrease in composite production when more PVA is present. This decrease in wet thickness is probably due to the viscosity change when the polymer is added, as shown before. ${ }^{3,36}$ The viscosity of the fermentation medium increased significantly after adding PVA, especially for the highest concentrations, thus decreasing the oxygen transfer rate and cell migration, and slowing down BC production rate. ${ }^{16,36}$ As long as PVA concentration is below $10 \% \mathrm{w} / \mathrm{v}$, we could harvest $\mathrm{BC} / \mathrm{PVA}$ composites with a wet thickness exceeding $4.0 \mathrm{~mm}$ after 10 days of fermentation, which is thick enough to perform mechanical testing.

The film shape and size are easily tailored by adjusting the shape and size of the fermentation vessel (Supporting Information Figure S7c-e). The entire fermentation step is carried out under mild conditions without using or generating any toxic chemicals. The fabrication process is scalable, green, and environmentally friendly.

Tensile Properties of the BC/PVA Composites. The microstructure of a material influences its mechanical performance. Honeycomb structures are commonly observed in nature, and are seen in many biological systems like wood, ${ }^{5}$ turtle shells, ${ }^{37}$ bamboo, ${ }^{38}$ and cork. ${ }^{39}$ The honeycomb microstructure of wood, which is formed through a different mechanism, ${ }^{5,40}$ is demonstrated to be one of the reasons that wood possesses excellent mechanical properties, ${ }^{27}$ hence the growing interest in mimicking this structure. ${ }^{41}$ The random fibrous structure of natural $\mathrm{BC}$, originating from the bacteria moving freely in all directions during fermentation, ${ }^{42}$ limits its mechanical properties. Therefore, the microscale spiral honeycomb structure observed here in the $10 \%$ PVA-BC-FT samples is likely to affect BC's mechanical properties. Tensile tests are performed to test this. The 10\% PVA-BC-FT samples show a significantly higher ultimate strength (314.98 $\pm 20.51 \mathrm{MPa}$, Figure 4a,b) and elongation at break (8.58 \pm $1.27 \%$, Figure $4 c$ ) compared to the other groups. Comparing the samples that have undergone the FT procedure to the ones 

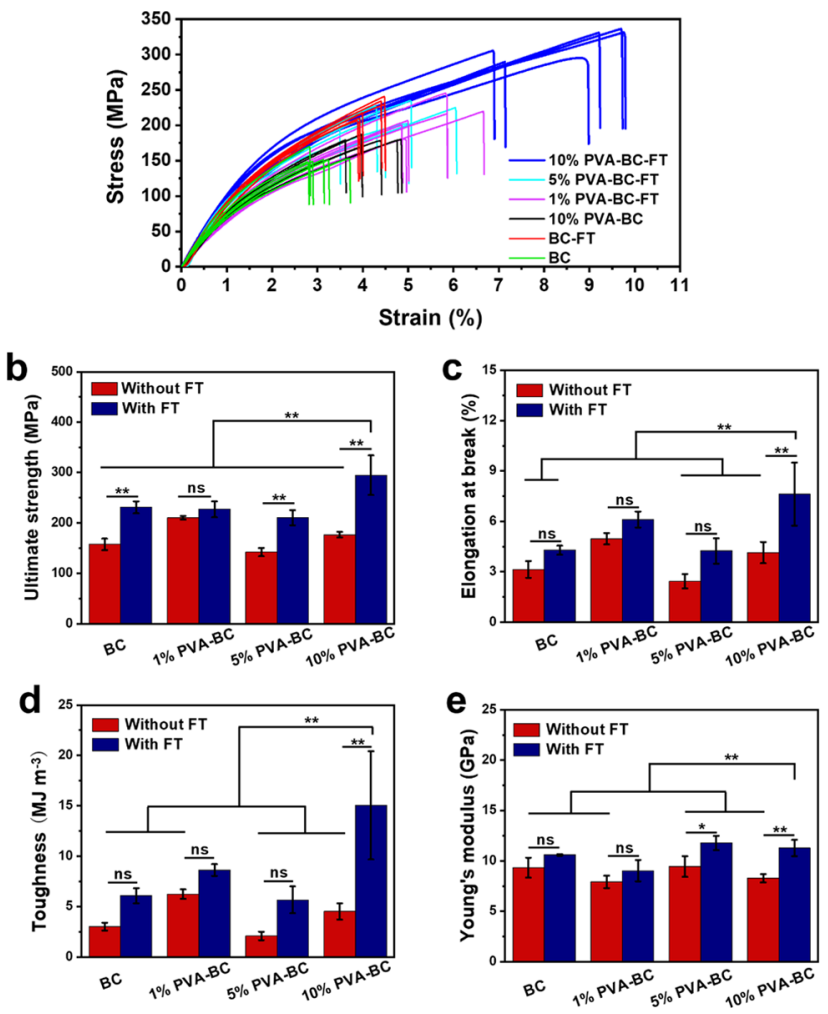

Figure 4. Tensile properties of the BC/PVA composite film with and without FT. (a) Stress-strain curves, (b) ultimate strength, (c) elongation at break, (d) toughness, and (e) Young's moduli. * $p<$ 0.05 , significant; $* * p<0.01$, significant; ns, insignificant.

which have not, we find that 10\% PVA-BC-FT and BC-FT (ultimate strength: $223.07 \pm 13.82 \mathrm{MPa}$; elongation at break: $4.19 \pm 0.29 \%)$ show higher ultimate strength and higher elongation at break than 10\% PVA-BC (ultimate strength: $176.66 \pm 5.59 \mathrm{MPa}$; elongation at break: $4.14 \pm 0.63 \%)$ and $\mathrm{BC}$ (ultimate strength: $153.89 \pm 8.21 \mathrm{MPa}$; elongation at break: $3.10 \pm 0.35 \%$ ); see Figure $4 \mathrm{~b}, \mathrm{c}$. This indicates that the FT procedure improves the tensile properties of $\mathrm{BC}$. The ultimate strengths of $1 \%$ PVA-BC-FT $(211.64 \pm 21.45 \mathrm{MPa}$, Figure $4 \mathrm{~b})$ and 5\% PVA-BC-FT $(217.41 \pm 13.96 \mathrm{MPa}$, Figure $4 \mathrm{~b})$ are close to that of BC-FT. Therefore, the main reason for this sharp increase in the ultimate strength of $10 \%$ PVA-BCFT is not the addition of PVA, but rather its different microstructure. Due to the contributions from a high tensile strength and a higher elongation at break, 10\% PVA-BC-FT shows a toughness $\left(17.76 \pm 3.63 \mathrm{MJ} \mathrm{m}^{-3}\right)$, which is significantly higher than that of $\mathrm{BC}\left(2.89 \pm 0.33 \mathrm{MJ} \mathrm{m}^{-3}\right.$,
Figure 4d). The Young's modulus of the 10\% PVA-BC-FT composite does not increase significantly compared to most other specimen types, with Young's moduli of all sample types varying between $7.41 \pm 0.85$ and $11.24 \pm 0.63 \mathrm{GPa}$ (Figure $4 \mathrm{e})$. In conclusion, the improved mechanical properties of $10 \%$ PVA-BC-FT are most likely linked to its nanofibrous layered structure and honeycomb microstructure. PVA was previously added to the fermentation medium to form BC/PVA composites. $^{33,43}$ In these studies, however, the maximum tensile strength was smaller (less than $55 \mathrm{MPa}$ ), different posttreatment methods were used, and no honeycomb structure was observed. Our BC/PVA composite film shows competitive tensile strength and toughness values in comparison with other BC-based composites fabricated under mild conditions (Supporting Information Table S1).

To further understand the failure mechanism of this new material, cross sections of the 10\% PVA-BC-FT specimens after tensile testing are imaged, showing fibrous de-spiral (Figure 5a-d and Supporting Information Figure S8) and pullout morphologies (Figure $5 \mathrm{e}-\mathrm{h}$ ). The pull-out morphology was previously reported in other layered materials and was suggested to be responsible for the toughness enhancement during failure. ${ }^{4-46}$ This failure process can absorb more energy under tension, thus could contribute to the high strength and toughness measured in this honeycomb nanofibrous material.

Finite-Element Simulations. To study the mechanistic aspects of how the spiral honeycomb structure improves BC mechanical properties, numerical simulations are carried out. In the random structure, a high level of localized stress concentrations is observed in some regions of the structure (Figure 6a). These regions are prone to failure for a higher level of applied strains. In contrast, the stress is more uniformly distributed in the spiral honeycomb structure (Figure 6b). Moreover, the maximum principal stress in the spiral honeycomb structure is lower than the maximum stress in the random structure $\left(\left(S_{\max }\right)_{\text {Spiral honeycomb }}\right)=2.22 \mathrm{GPa}$, $\left.\left(S_{\max }\right)_{\text {Random }}=4.81 \mathrm{GPa}\right)$ when both structures are subjected to the same level of axial strain. The computational models are limited to the elastic regime and we do not include any plasticity and post-yielding in the model. Nevertheless, the computational models confirm that the structures with random networks tend to break at a lower strain level due to the inhomogeneous stress distribution throughout the structure compared to spiral honeycomb lattice structures. Moreover, the predicted numerical elastic moduli (slope of curves in Figure 6c) agree with the experimental observations, showing higher elastic modulus for the structures with spiral honeycomb lattices given the fact that both models had similar
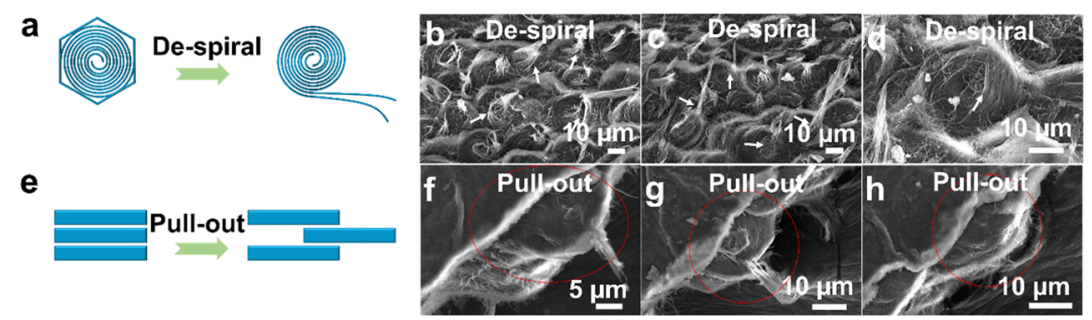

Figure 5. De-spiral and pull-out breaking mechanisms of the bacterial cellulose films with honeycomb microstructure. Schematics of (a) de-spiral and (e) pull-out; SEM images of the $(\mathrm{b}-\mathrm{d})$ de-spiral morphology (white arrows) and $(\mathrm{f}-\mathrm{h}$ ) pull-out morphology (encircled in red) of the honeycomb $\mathrm{BC}$ film after tensile testing. 

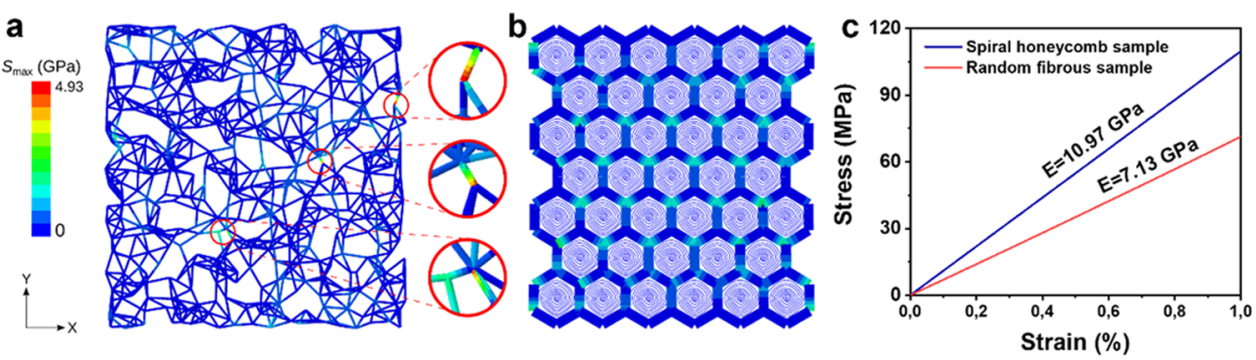

Figure 6. Numerical simulation results of the BC film with honeycomb and random microstructure. Stress distribution in (a) random structure and (b) spiral honeycomb structure. (c) Stress-strain curve of random and spiral honeycomb structures predicted by FE simulations.

overall densities. The small differences between the numerical and experimental results could be due to the simplifications considered for the simulation of these intricate structures.

\section{CONCLUSIONS}

The natural biopolymer bacterial cellulose possesses an organized fibril structure at the nanoscale. At the microscale, however, the $\mathrm{BC}$ nanofibers are distributed randomly. To further increase the tensile strength and toughness of $\mathrm{BC}$, it is important but still remains challenging to control the organization of $\mathrm{BC}$ at the microscale. Here, we demonstrate the combination of an in situ biofabrication of BC with $10 \% \mathrm{w} /$ $\mathrm{v}$ PVA with a post-treatment procedure including freezingthawing, boiling, washing, and air-drying, to generate a strong and tough $\mathrm{BC}$ film with a highly organized spiral nanofibrous honeycomb microstructure. At high enough concentration, PVA likely affects the hydrogen-bond network of BC fibrils. The weakened BC fibers could therefore self-assemble in the observed spirals with a highly organized hexagonal microunit. As a result, this honeycomb $\mathrm{BC}$ film shows a $2 \times$ increase (from $154 \mathrm{MPa}$ for $\mathrm{BC}$ to $315 \mathrm{MPa}$ for honeycomb $\mathrm{BC}$ ) in tensile strength and a $5 \times$ increase (from $2.9 \mathrm{MJ} \mathrm{m}^{-3}$ for $\mathrm{BC}$ to 17.8

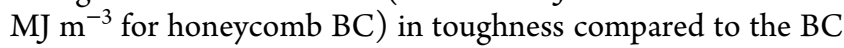
samples with random nanofibrous structure. The sharp increase in mechanical properties is due to this special honeycomb structure, because materials of similar composition with no honeycomb structure show reduced tensile strength and toughness. In addition to experiments, finite-element simulations also indicate improved mechanical properties resulting from the honeycomb structure. Furthermore, shape, size, and thickness of this material are controllable by simply adjusting the shape and size of the culture vessel and the cultivation time. This fabrication method provides a green and mild platform for incorporating beneficial polymers into $\mathrm{BC}$ to produce materials with superior mechanical properties and complex biomimetic structures on a large scale.

\section{EXPERIMENTAL SECTION}

Materials, Strain, and Culture Conditions. Poly(vinyl alcohol) (PVA, $M_{\mathrm{w}} 89000-98000$, >99\% hydrolyzed), tryptone (Pancreatic digest of casein), yeast extract, agar, citric acid monohydrate (ACS reagent, $\geq 99.0 \%$ ), and cellulase from Trichoderma reesei (aqueous solution, $\geq 700$ units $^{-1}$ ) were purchased from Sigma-Aldrich. $\mathrm{D}(+)$ Glucose monohydrate, sodium chloride $(\mathrm{NaCl})$, and disodium hydrogen phosphate $(\geq 99.0 \%)$ were obtained from Carl Roth GmbH.

The cellulose producing strain Gluconacetobacter hansenii (ATCC 53582 ) was propagated in Hestrin-Schramm (HS) medium (5.0 $\mathrm{g} \mathrm{L}^{-1}$ tryptone, $5.0 \mathrm{~g} \mathrm{~L}^{-1}$ yeast extract, $2.7 \mathrm{~g} \mathrm{~L}^{-1}$ disodium hydrogen phosphate, $1.5 \mathrm{~g} \mathrm{~L}^{-1}$ citric acid, and $20 \mathrm{~g} \mathrm{~L}^{-1}$ glucose) at $30{ }^{\circ} \mathrm{C}$ under static conditions for 3 days to obtain the $\mathrm{BC}$ pellicle. The inoculum for bacterial fermentation was prepared by treating the $\mathrm{BC}$ pellicle with cellulase at $180 \mathrm{rpm}$ at $30{ }^{\circ} \mathrm{C}$ overnight. The solution was then centrifuged $\left(4{ }^{\circ} \mathrm{C}, 3220 \mathrm{~g}\right.$ centrifuge speed, $\left.10 \mathrm{~min}\right)$ to remove the cellulase, and the bacterial pellet was resuspended in fresh HS medium to obtain an $\mathrm{OD}_{600}$ of 1 . We then used $1 \% \mathrm{v} / \mathrm{v}$ of this solution as the inoculum.

Growing Composite Materials by Bacteria. PVA powder was dissolved into HS medium at concentrations of 1,5 , and $10 \% \mathrm{w} / \mathrm{v}$ followed by boiling in a kitchen microwave oven for $5 \mathrm{~min}$ and cooling. This process was repeated three times to sterilize the solution. After that, the polymer/medium solution was inoculated with G. hansenii bacteria (Gluconacetobacter hansenii ATCC 53582 bacteria were obtained from the American Type Culture Collection (ATCC)). Fermentation was then carried out at $30{ }^{\circ} \mathrm{C}$ for 10 days under static conditions. The nanofibrous polymer composites were formed at the air-liquid interface such that the shape of the composite pellicle was determined by the shape of the fermentation chamber (flask or rectangular box-like) and the material thickness was established by the culture time.

Post-Treatment of the Nanofibrous Composites. After fermentation, the freshly formed solid pellicle was transferred to a plastic Petri dish and treated with a "freezing-thawing" method. Briefly, the solid pellicle was stored at $-20{ }^{\circ} \mathrm{C}$ to be frozen for $24 \mathrm{~h}$ and was, then, left to thaw at room temperature for $6 \mathrm{~h}$. The PVA inside the composites is known to cross-link by the crystals formed after repeating this "freezing-thawing" procedure for five times. ${ }^{26}$ After cross-linking, the pellicles were boiled to kill the bacteria and washed with distilled water for 3 days to remove the unreacted polymers and impurities, followed by drying in the air to form the final polymer/cellulose nanofibrous composite films.

Characterization of the BC/PVA Nanofibrous Composites. The material morphology was observed by scanning electron microscopy (SEM, JEOL JSM 6010 LA). The material was sputtercoated with gold-palladium at $20 \mathrm{~mA}$ for $60 \mathrm{~s}$ and was observed at 5$15 \mathrm{kV}$ under vacuum. SEM was carried out on the specimens after the boiling and washing steps.

To check for the presence of polymers inside the composites, FTIR (PerkinElmer, Spectrum 100) equipped with an attenuated total reflection (ATR) accessory was used. The FTIR spectra were the average of 20 scans in the $550-4000 \mathrm{~cm}^{-1}$ range at a resolution of 4 $\mathrm{cm}^{-1}$.

TGA (Mettler Toledo) was assessed at $30-1000{ }^{\circ} \mathrm{C}$ with a heating rate of $10{ }^{\circ} \mathrm{C} \mathrm{min}-1$ in the air atmosphere. Derivative thermogravimetry (DTG), the first derivative of the TGA curve, was also plotted (Supporting Information Figure S6b).

The tensile tests were performed using a Zwick/Roell Z010 universal testing machine with a $500 \mathrm{~N}$ load cell and $1 \mathrm{kN}$ grips. The measuring distance between the clamps was $10 \mathrm{~mm}$, and the samples were tested with a loading rate of $2 \mathrm{~mm} / \mathrm{min}$. At least six specimens per group were measured for the data presented here.

Measuring the Bacterial Viability and the Yield of the Composites. The effects of adding PVA on the bacterial viability was assessed with the colony-forming unit (CFU) measurements. Briefly, G. hansenii with/without added PVA was cultured for 0, 5, 10, and 15 days statically at $30{ }^{\circ} \mathrm{C}$. After the respective incubation times, the cellulase treatment was carried out by adding cellulase and incubating the resulting mixture at $30{ }^{\circ} \mathrm{C}$ overnight at $180 \mathrm{rpm}$. Then, the treated 
solutions were centrifuged to remove the cellulase, medium, and PVA. The bacterial pellet was resuspended in the same initial volume of saline $(0.9 \% \mathrm{w} / \mathrm{v} \mathrm{NaCl})$. Dilutions of this in the range of $10^{0}-10^{-8}$ were made and $20 \mu \mathrm{L}$ of each dilution was spotted on HS agar plates (supplemented with $2 \% \mathrm{v} / \mathrm{v}$ acetic acid). The plates were then incubated at $30{ }^{\circ} \mathrm{C}$ for 3 days, and the number of colonies was enumerated and the $\log _{10}(\mathrm{CFU} / \mathrm{mL})$ was calculated.

The yield of the composite materials was assessed by measuring the wet thicknesses of the different composite pellicle specimens (of varying culturing times) with a Vernier caliper. The wet and dried sample weights were measured using a weighing balance.

Finite-Element Simulations. For numerical simulation, a nonlinear finite element (FE) solver (Abaqus Standard 6.14) was used. The geometry of the random structures was created in Matlab (R2018b) software and was then imported as an input file into Abaqus software. We used the quadratic Timoshenko beam element (B22) since these elements allow for axial deformations, bending, and shear. Each nanofiber was simulated as a beam with a circular cross section and a diameter of $0.059 \mathrm{~mm}$. Each strut of the honeycomb unit cell was assumed to consist of 5000 parallel nanofibers and to have a rectangular cross section with a width $(W)$ of $0.059 \mathrm{~mm}$ and a length $(L)$ of $5 \mathrm{~mm}$. Therefore, the out-of-plane thickness $(T)$ of both structures was considered $0.059 \mathrm{~mm}$. The dimensions $(W \times H)$ of the random structure and spiral honeycomb were considered to be $8 \times 8$ and $138 \times 138 \mathrm{~mm}^{2}$, respectively. The level of connectivity of the random structure, which was defined as the average connectivity of all nodes, ${ }^{47}$ was considered 5.5. This value was selected in a way that both random structure and honeycomb have equal densities.

An elastic material model was used for both structures $(E=125$ $\mathrm{GPa}$ and $\nu=0.2$ ). The appropriate values for elastic properties were obtained via calibration, and the initial range for Young's moduli was selected based on previous studies. ${ }^{18,48}$ In both models, a uniaxial displacement-controlled stretch test in the $y$ direction (Figure $6 \mathrm{a}, \mathrm{b}$ ) was simulated. To this aim, two reference points were defined on the top and bottom of the structure, which were kinematically coupled with their corresponding nodes at the top and bottom of the structure. A displacement boundary condition corresponding to $1 \%$ strain was applied to the top reference point while all degrees of freedom of the bottom reference point were constrained. The normal stress, $\sigma=F / A$, was defined as the ratio of the reaction force, $F$, to the initial cross-sectional area, $A=W \times T$.

Statistics. Statistical analyses were performed on https://astatsa. $\mathrm{com} /$. The experimental groups were compared using one-way (single factor) ANOVA with post-hoc Tukey's HSD (honest significant difference) tests.

\section{ASSOCIATED CONTENT}

\section{s) Supporting Information}

The Supporting Information is available free of charge at https://pubs.acs.org/doi/10.1021/acsami.0c15886.

SEM, FTIR, TGA, yield, and optical images of the honeycomb and control samples, and comparison of tensile strength and toughness of cellulose-based film materials (PDF)

\section{AUTHOR INFORMATION}

\section{Corresponding Author}

Marie-Eve Aubin-Tam - Department of Bionanoscience, Kavli Institute of Nanoscience, Delft University of Technology, 2629 HZ Delft, The Netherlands; O orcid.org/0000-0001-99952623; Email: M.E.Aubin-Tam@tudelft.nl

\section{Authors}

Kui Yu - Department of Bionanoscience, Kavli Institute of Nanoscience, Delft University of Technology, 2629 HZ Delft, The Netherlands
Srikkanth Balasubramanian - Department of Bionanoscience, Kavli Institute of Nanoscience, Delft University of Technology, 2629 HZ Delft, The Netherlands

Helda Pahlavani - Department of Biomechanical Engineering, Faculty of Mechanical, Maritime, and Materials Engineering, Delft University of Technology, 2628 CD Delft, The Netherlands

Mohammad J. Mirzaali - Department of Biomechanical Engineering, Faculty of Mechanical, Maritime, and Materials Engineering, Delft University of Technology, 2628 CD Delft, The Netherlands

Amir A. Zadpoor - Department of Biomechanical Engineering, Faculty of Mechanical, Maritime, and Materials Engineering, Delft University of Technology, 2628 CD Delft, The Netherlands; (1) orcid.org/0000-0003-3234-2112

Complete contact information is available at: https://pubs.acs.org/10.1021/acsami.0c15886

\section{Notes}

The authors declare no competing financial interest.

\section{ACKNOWLEDGMENTS}

The authors thank Tessa Essers and Mascha Slingerland for their help with the tensile testing, Marlies Nijemeisland for assistance with FTIR spectroscopy, and Bart van der Linden for the TGA measurements. They thank Ramon van der Valk and Roland Kieffer for their suggestions to this manuscript and their lab support, and Ewa Spiesz, Dominik Schmieden, Anne S. Meyer, and Yuemei Lin for their advice. K.Y. is funded by the China Scholarship Council (CSC no. 201706630001). S.B. is funded by the Air Force Office of Scientific Research, Asian Office of Aerospace Research and Development (grant no. FA2386-18-1-4059).

\section{REFERENCES}

(1) Wegst, U. G.; Bai, H.; Saiz, E.; Tomsia, A. P.; Ritchie, R. O. Bioinspired Structural Materials. Nat. Mater. 2015, 14, 23-36.

(2) Wang, Q.; Schniepp, H. C. Strength of Recluse Spider's Silk Originates from Nanofibrils. ACS Macro Lett. 2018, 7, 1364-1370.

(3) Barthelat, F. Nacre from Mollusk Shells: A Model for HighPerformance Structural Materials. Bioinspiration Biomimetics 2010, 5, No. 035001.

(4) Armiento, A. R.; Hatt, L. P.; Rosenberg, G. S.; Thompson, K.; Stoddart, M. J. Functional Biomaterials for Bone Regeneration: A Lesson in Complex Biology. Adv. Funct. Mater. 2020, No. 1909874.

(5) Gibson, L. J. The Hierarchical Structure and Mechanics of Plant Materials. J. R. Soc. Interface 2012, 9, 2749-2766.

(6) Huang, W.; Restrepo, D.; Jung, J. Y.; Su, F. Y.; Liu, Z.; Ritchie, R. O.; McKittrick, J.; Zavattieri, P.; Kisailus, D. Multiscale Toughening Mechanisms in Biological Materials and Bioinspired Designs. Adv. Mater. 2019, 31, No. 1901561.

(7) Spiesz, E. M.; Schmieden, D. T.; Grande, A. M.; Liang, K.; Schwiedrzik, J.; Natalio, F.; Michler, J.; Garcia, S. J.; Aubin-Tam, M. E.; Meyer, A. S. Bacterially Produced, Nacre-Inspired Composite Materials. Small 2019, 15, No. 1805312.

(8) Yu, S.-H.; Wu, H.-A.; Liu, C.; Wen, S.-M.; Meng, Y.-F.; Pan, Z.; Chen, S.-M.; Zhu, Y.-B.; Cui, C.; Zhao, R.; Gao, H.-L. Bioinspired Hierarchical Helical Nanocomposite Macrofibers Based on Bacterial Cellulose Nanofibers. Natl. Sci. Rev. 2020, 7, 73-83.

(9) Moradali, M. F.; Rehm, B. H. A. Bacterial Biopolymers: From Pathogenesis to Advanced Materials. Nat. Rev. Microbiol. 2020, 18, 195-210.

(10) Malho, J. M.; Laaksonen, P.; Walther, A.; Ikkala, O.; Linder, M. B. Facile Method for Stiff, Tough, and Strong Nanocomposites by 
Direct Exfoliation of Multilayered Graphene into Native Nanocellulose Matrix. Biomacromolecules 2012, 13, 1093-1099.

(11) Chen, Y.; Fu, J.; Dang, B.; Sun, Q.; Li, H.; Zhai, T. Artificial Wooden Nacre: A High Specific Strength Engineering Material. ACS Nano 2020, 14, 2036-2043.

(12) Mao, L. B.; Gao, H. L.; Yao, H. B.; Liu, L.; Colfen, H.; Liu, G.; Chen, S. M.; Li, S. K.; Yan, Y. X.; Liu, Y. Y.; Yu, S. H. Synthetic Nacre by Predesigned Matrix-Directed Mineralization. Science 2016, 354, 107-110.

(13) Wei, P.; Cai, J.; Zhang, L. High-Strength and Tough Crystalline Polysaccharide-Based Materials. Chin. J. Chem. 2020, 38, 761-771.

(14) Florea, M.; Reeve, B.; Abbott, J.; Freemont, P. S.; Ellis, T. Genome Sequence and Plasmid Transformation of the Model HighYield Bacterial Cellulose Producer Gluconacetobacter Hansenii ATCC 53582. Sci. Rep. 2016, 6, No. 23635.

(15) Mittal, N.; Ansari, F.; Gowda, V. K.; Brouzet, C.; Chen, P.; Larsson, P. T.; Roth, S. V.; Lundell, F.; Wagberg, L.; Kotov, N. A.; Soderberg, L. D. Multiscale Control of Nanocellulose Assembly: Transferring Remarkable Nanoscale Fibril Mechanics to Macroscale Fibers. ACS Nano 2018, 12, 6378-6388.

(16) Wang, J.; Tavakoli, J.; Tang, Y. Bacterial Cellulose Production, Properties and Applications with Different Culture Methods - A Review. Carbohydr. Polym. 2019, 219, 63-76.

(17) Wu, Z. Y.; Liang, H. W.; Chen, L. F.; Hu, B. C.; Yu, S. H. Bacterial Cellulose: A Robust Platform for Design of Three Dimensional Carbon-Based Functional Nanomaterials. Acc. Chem. Res. 2016, 49, 96-105.

(18) Wang, S.; Jiang, F.; Xu, X.; Kuang, Y.; Fu, K.; Hitz, E.; Hu, L. Super-Strong, Super-Stiff Macrofibers with Aligned, Long Bacterial Cellulose Nanofibers. Adv. Mater. 2017, 29, No. 1702498.

(19) Gao, M.; Li, J.; Bao, Z.; Hu, M.; Nian, R.; Feng, D.; An, D.; Li, X.; Xian, M.; Zhang, H. A Natural In Situ Fabrication Method of Functional Bacterial Cellulose Using a Microorganism. Nat. Commun. 2019, 10, No. 437

(20) Yao, J.; Chen, S.; Chen, Y.; Wang, B.; Pei, Q.; Wang, H. Macrofibers with High Mechanical Performance Based on Aligned Bacterial Cellulose Nanofibers. ACS Appl. Mater. Interfaces 2017, 9, 20330-20339.

(21) Gutierrez, E.; Burdiles, P. A.; Quero, F.; Palma, P.; Olate-Moya, F.; Palza, H. 3D Printing of Antimicrobial Alginate/BacterialCellulose Composite Hydrogels by Incorporating Copper Nanostructures. ACS Biomater. Sci. Eng. 2019, 5, 6290-6299.

(22) Guan, Q.-F.; Han, Z.-M.; Luo, T.-T.; Yang, H.-B.; Liang, H.-W.; Chen, S.-M.; Wang, G.-S.; Yu, S.-H. A General Aerosol-Assisted Biosynthesis of Functional Bulk Nanocomposites. Natl. Sci. Rev. 2019, 6, 64-73.

(23) Wang, S.; Li, T.; Chen, C.; Kong, W.; Zhu, S.; Dai, J.; Diaz, A. J.; Hitz, E.; Solares, S. D.; Li, T.; Hu, L. Transparent, Anisotropic Biofilm with Aligned Bacterial Cellulose Nanofibers. Adv. Funct. Mater. 2018, 28, No. 1707491.

(24) Wu, Z.; Chen, S.; Wu, R.; Sheng, N.; Zhang, M.; Ji, P.; Wang, H. Top-Down Peeling Bacterial Cellulose to High Strength Ultrathin Films and Multifunctional Fibers. Chem. Eng. J. 2020, 391, No. 123527.

(25) Wahid, F.; Wang, F. P.; Xie, Y. Y.; Chu, L. Q.; Jia, S. R.; Duan, Y. X.; Zhang, L.; Zhong, C. Reusable Ternary PVA Films Containing Bacterial Cellulose Fibers and Epsilon-Polylysine with Improved Mechanical and Antibacterial Properties. Colloids Surf., B 2019, 183, No. 110486.

(26) Osorio, M.; Velásquez-Cock, J.; Restrepo, L. M.; Zuluaga, R.; Gañán, P.; Rojas, O. J.; Ortiz-Trujillo, I.; Castro, C. Bioactive 3DShaped Wound Dressings Synthesized from Bacterial Cellulose: Effect on Cell Adhesion of PolyVinyl Alcohol Integrated In Situ. Int. J. Polym. Sci. 2017, 2017, No. 3728485.

(27) Zhang, Q.; Yang, X.; Li, P.; Huang, G.; Feng, S.; Shen, C.; Han, B.; Zhang, X.; Jin, F.; Xu, F.; Lu, T. J. Bioinspired Engineering of Honeycomb Structure- Using Nature to Inspire Human Innovation. Prog. Mater. Sci. 2015, 74, 332-400.
(28) Rehman, M. U.; Rojas, J. P. Optimization of Compound Serpentine- Spiral Structure for Ultra-Stretchable Electronics. Extreme Mech. Lett. 2017, 15, 44-50.

(29) Si, X.; Lu, F.; Chen, J.; Lu, R.; Huang, Q.; Jiang, H.; Taarning, E.; Xu, J. A Strategy for Generating High-Quality Cellulose and Lignin Simultaneously from Woody Biomass. Green Chem. 2017, 19, 48494857.

(30) Khakalo, A.; Tanaka, A.; Korpela, A.; Orelma, H. Delignification and Ionic Liquid Treatment of Wood toward Multifunctional High-Performance Structural Materials. ACS Appl. Mater. Interfaces 2020, 12, 23532-23542.

(31) Ma, L.; Bi, Z.; Xue, Y.; Zhang, W.; Huang, Q.; Zhang, L.; Huang, Y. Bacterial Cellulose: An Encouraging Eco-Friendly NanoCandidate for Energy Storage and Energy Conversion. J. Mater. Chem. A 2020, 8, 5812-5842.

(32) Ray, D.; Sain, S. In Situ Processing of Cellulose Nanocomposites. Composites, Part A 2016, 83, 19-37.

(33) Castro, C.; Vesterinen, A.; Zuluaga, R.; Caro, G.; Filpponen, I.; Rojas, O. J.; Kortaberria, G.; Gañán, P. In Situ Production of Nanocomposites of Poly(Vinyl Alcohol) and Cellulose Nanofibrils from Gluconacetobacter Bacteria: Effect of Chemical Crosslinking. Cellulose 2014, 21, 1745-1756.

(34) Yokoyama, F.; Masada, I.; Shimamura, K.; Ikawa, T.; Monobe, K. Morphology and Structure of Highly Elastic Poly(Vinyl Alcohol) Hydrogel Prepared by Repeated Freezing-and-Melting. Colloid Polym. Sci. 1986, 264, 595-601.

(35) Ruka, D. R.; Simon, G. P.; Dean, K. M. Altering the Growth Conditions of Gluconacetobacter Xylinus to Maximize the Yield of Bacterial Cellulose. Carbohydr. Polym. 2012, 89, 613-622.

(36) Chen, Y.; Zhou, X.; Lin, Q.; Jiang, D. Bacterial Cellulose/ Gelatin Composites: In Situ Preparation and Glutaraldehyde Treatment. Cellulose 2014, 21, 2679-2693.

(37) Naleway, S. E.; Porter, M. M.; McKittrick, J.; Meyers, M. A. Structural Design Elements in Biological Materials: Application to Bioinspiration. Adv. Mater. 2015, 27, 5455-5476.

(38) Chen, C.; Li, Z.; Mi, R.; Dai, J.; Xie, H.; Pei, Y.; Li, J.; Qiao, H.; Tang, H.; Yang, B.; Hu, L. Rapid Processing of Whole Bamboo with Exposed, Aligned Nanofibrils toward a High-Performance Structural Material. ACS Nano 2020, 14, 5194-5202.

(39) Miranda, I.; Gominho, J.; Pereira, H. Cellular Structure and Chemical Composition of Cork from the Chinese Cork Oak (Quercus Variabilis). J. Wood Sci. 2013, 59, 1-9.

(40) Wodzicki, T. J. Natural Factors Affecting Wood Structure. Wood Sci. Technol. 2001, 35, 5-26.

(41) Yu, Z. L.; Qin, B.; Ma, Z. Y.; Gao, Y. C.; Guan, Q. F.; Yang, H. B.; Yu, S. H. Emerging Bioinspired Artificial Woods. Adv. Mater. 2020, No. 2001086.

(42) Rahman, M. M.; Netravali, A. N. Aligned Bacterial Cellulose Arrays as "Green" Nanofibers for Composite Materials. ACS Macro Lett. 2016, 5, 1070-1074.

(43) Castro, C.; Zuluaga, R.; Rojas, O. J.; Filpponen, I.; Orelma, H.; Londoño, M.; Betancourt, S.; Gañán, P. Highly Percolated Poly(Vinyl Alcohol) and Bacterial Nanocellulose Synthesized In Situ by PhysicalCrosslinking: Exploiting Polymer Synergies for Biomedical Nanocomposites. RSC Adv. 2015, 5, 90742-90749.

(44) Wang, J.; Cheng, Q.; Lin, L.; Jiang, L. Synergistic Toughening of Bioinspired Poly(Vinyl Alcohol)-Clay-Nanofibrillar Cellulose Artificial Nacre. ACS Nano 2014, 8, 2739-2745.

(45) Zhao, H.; Yue, Y.; Zhang, Y.; Li, L.; Guo, L. Ternary Artificial Nacre Reinforced by Ultrathin Amorphous Alumina with Exceptional Mechanical Properties. Adv. Mater. 2016, 28, 2037-2042.

(46) Yan, Y.-X.; Yao, H.-B.; Yu, S.-H. Nacre-Like Ternary Hybrid Films with Enhanced Mechanical Properties by Interlocked Nanofiber Design. Adv. Mater. Interfaces 2016, 3, No. 1600296.

(47) Mirzaali, M. J.; Pahlavani, H.; Zadpoor, A. A. Auxeticity and Stiffness of Random Networks: Lessons for the Rational Design of 3D Printed Mechanical Metamaterials. Appl. Phys. Lett. 2019, 115, No. 021901. 
(48) Walker, K. T.; Goosens, V. J.; Das, A.; Graham, A. E.; Ellis, T. Engineered Cell-to-Cell Signalling within Growing Bacterial Cellulose Pellicles. Microb. Biotechnol. 2019, 12, 611-619. 